\title{
Screening and validation of plasma long non-coding RNAs as biomarkers for the early diagnosis and staging of oral squamous cell carcinoma
}

\author{
HONGCHENG JIA ${ }^{1 *}$, XUAN WANG ${ }^{2 *}$ and ZHENG SUN ${ }^{3}$ \\ ${ }^{1}$ Department of Stomatology, Beijing Ditan Hospital, Capital Medical University, Beijing 100015; \\ ${ }^{2}$ Department of Stomatology, Beijing Tongren Hospital, Capital Medical University, Beijing 100730; \\ ${ }^{3}$ Department of Oral Medicine, Beijing Stomatological Hospital, Capital Medical University, Beijing 100050, P.R. China
}

Received July 31, 2020; Accepted December 9, 2020

DOI: 10.3892/ol.2021.12433

\begin{abstract}
Oral squamous cell carcinoma (OSCC), characterized by a high recurrence rate, a poor prognosis and high morbidity, is the most prevalent malignancy of the oral cavity. The aberrant expression of long non-coding RNAs (lncRNAs) may lead to the development of various diseases, including cancer. Delayed diagnosis is the main reason for the poor prognosis. Therefore, the present study aimed to investigate the differential expression profiles of plasma lncRNAs in OSCC in order to screen target lncRNAs as biomarkers for the early diagnosis and staging of OSCC. The expression profiles of IncRNAs and mRNAs in OSCC were analyzed by microarray analysis. A total of 14 candidate lncRNAs were selected and analyzed using reverse transcription-quantitative polymerase chain reaction (RT-qPCR) using the array homologous samples. Subsequently, 4 target lncRNAs were measured by RT-qPCR in a large cohort, including 28 cases with TNM I/II [early-stage squamous cell carcinoma (ESCC) group], 36 cases with TNM III/IV [advanced-stage squamous cell carcinoma (ASCC) group], 16 cases with dysplasia [oral premalignant lesion (OPL) group] and 16 healthy controls (H group). Receiver operating characteristic (ROC) curves and logistic regression analysis were used to evaluate the diagnostic effects of the combined lncRNAs. In total, 6,606
\end{abstract}

Correspondence to: Professor Zheng Sun, Department of Oral Medicine, Beijing Stomatological Hospital, Capital Medical University, 4 Tiantanxili, Dongcheng, Beijing 100050, P.R. China E-mail: sunzheng12@vip.126.com

Dr Hongcheng Jia, Department of Stomatology, Beijing Ditan Hospital, Capital Medical University, 8 Jingshundongjie, Chaoyang, Beijing 100015, P.R. China

E-mail: jiahongcheng@ccmu.edu.cn

*Contributed equally

Key words: oral squamous cell carcinoma, oral premalignant lesions, long non-coding RNA, biomarker, microarray differentially expressed lncRNAs and 4,196 mRNAs were identified in OSCC. The expression trend of the 14 candidate IncRNAs was consistent with the microarray data. The expression level of ENST00000412740, NR_131012, ENST00000588803 and NR_038323 exhibited significant differences in the H, OPL, ESCC and ASCC groups $(\mathrm{P}<0.05)$. ROC curve and logistic regression analyses revealed that the diagnostic efficacy of the combined lncRNAs was more prominent than that of a single lncRNA, particularly in the ESCC and ASCC groups. In conclusion, the present study identified the differential expression profiles of plasma IncRNAs in OSCC and demonstrated that ENST00000412740, NR_131012, ENST00000588803 and NR_038323 may be promising biomarkers for the early diagnosis and staging of OSCC. These findings may provide novel targets for the early diagnosis and staging of OSCC, which may provide an objective basis for clinical decision-making.

\section{Introduction}

Oral squamous cell carcinoma (OSCC) is the most prevalent malignancy of the oral cavity, accounting for $>90 \%$ of oral cancer cases, with 354,864 estimated new cases and 177,384 mortalities worldwide in $2018(1,2)$. OSCC, characterized by a high recurrence, a poor prognosis and high morbidity, severely affects the quality of life of patients. Therefore, OSCC poses a burden to global health. OSCC is preceded in $67 \%$ of cases by oral premalignant lesions (OPLs), of which 1-18\% undergo malignant transformation into OSCC (3). Patients with early-stage squamous cell carcinoma (ESCC; TNM I and II) survive longer than those with advanced-stage squamous cell carcinoma (ASCC; TNM III and IV), with survival rates of 64.2 and $30.1 \%$ for early and late stages, respectively (4). Despite improvements in treatment modalities, the 5-year overall survival rate has improved only marginally, with $33 \%$ of cases surviving between 1973 and 2014, compared with 41\% between 2006-2011 (5,6). Delayed diagnosis and the lack of accurate and timely treatment, derived from the bias of the standards of clinical decision-making based on the clinical experience and subjective judgment of doctors, are considered to be the major reasons for the poor prognosis. A minimally 
invasive, reliable and sensitive marker is urgently required to provide an objective basis for clinical decision-making.

Long non-coding RNAs (lncRNAs) are a class of RNA molecules with transcripts of $>200$ nucleotides in lengths, which were first discovered as 'transcriptional noise' in 1989. However, increasing evidence has suggested that lncRNAs are involved in gene expression regulations at the epigenetic, transcriptional and post-transcriptional levels, and are essential in physiological events (7-9). The aberrant expression of lncRNAs may directly or indirectly lead to the development of various diseases, including cancer $(10,11)$. IncRNAs may be promising biomarkers in cancer diagnosis and prognosis $(12,13)$. For example, MALAT1 may be used as a marker for the early diagnosis of prostate cancer (14), the upregulation of HOTAIR expression is indicative of a poor prognosis in colon and breast cancer (15), and the downregulation of GAS5 expression is indicative of a poor prognosis in gastric cancer (16). IncRNAs have also been revealed to be differentially expressed in tissues and salivary samples of the normal oral mucosa, in OPLs and OSCC (17-20). However, to the best of our knowledge, the differential expression profiles of IncRNAs in the plasma of patients with OSCC has not yet been reported.

In the present study, the differential expression profiles of plasma lncRNAs in OSCC were first investigated using microarray analysis to screen candidate lncRNAs, followed by reverse transcription-quantitative polymerase chain reaction (RT-qPCR) analysis. Subsequently, the target lncRNAs were further validated by RT-qPCR to estimate the diagnostic efficacy of plasma lncRNAs from patients with OPL, ESCC and ASCC. The results of the present study may provide novel targets for the early diagnosis and staging of OSCC, which may also provide an objective basis for clinical decision-making for the early diagnosis, reasonable implementation of the treatment plan and prognosis evaluation of OSCC.

\section{Materials and methods}

Samples. A total of 67 patients with OSCC (39 men and 28 women; age range, 47-75 years; mean age, 63.5 years), 16 patients with OPL (3 cases of mild dysplasia, 7 cases of moderate dysplasia and 6 cases of severe dysplasia) and 19 healthy control individuals (H group) were recruited between December 2013 and May 2015 from Capital Medical University Beijing Stomatological Hospital. A total of three patients with TNM III/IV OSCC and 3 healthy controls were selected for microarray analysis (Table SI), and the remaining samples were used for PCR validation, including 64 patients with OSCC (39 males and 25 females; age range, 47-75 years; mean age, 63.3 years; TNM staging is presented in Table SII), 16 patients with OPLs (9 males and 7 females; age range, 43-72 years; mean age, 58.1 years; all with epithelial dysplasia) and 16 healthy controls ( 8 males and 8 females; age range, 37-61 years; mean age, 48.6 years). The recruited subjects had no medical history of other types of cancer. Blood samples were collected in vacuum tubes with EDTA anticoagulant and were isolated by centrifugation at $1,000 \mathrm{x}$ g for $10 \mathrm{~min}$ at $4^{\circ} \mathrm{C}$ to obtain the plasma. The collected plasma was stored at $-80^{\circ} \mathrm{C}$ into a separate RNase-free tube prior to further analysis, which was divided into 400-500 $\mu \mathrm{l} /$ tubes. Blood samples with hemolysis were excluded and samples with absence of hemolysis were included.
The present study was approved by the Ethics Committee of the Capital Medical University Beijing Stomatological Hospital (Beijing, China; approval no. 201314), and written informed consent was provide by all participants prior to the study start.

Microarray assay. The differential expression profiles of lncRNAs and mRNAs in the frozen plasma of patients with OSCC were analyzed by KangChen Biotechnology Co., Ltd. using Arraystar Human LncRNA Microarray V4.0 (Agilent Technologies, Inc.). Total RNA was extracted from $400 \mu \mathrm{l}$ plasma using TRIzol $^{\circledR}$ reagent (Invitrogen; Thermo Fisher Scientific, Inc.), purified using the RNasey Mini kit (Qiagen, Inc.), and amplified and labeled using the Quick Amp Labeling kit One-Color (Agilent Technologies, Inc.), according to the manufacturer's protocol. The aforementioned steps were repeated until the cRNA production was $>1.65 \mu \mathrm{g}$ and the specific activity was $>9.0 \mathrm{pmol} \mathrm{Cy} 3 / \mu \mathrm{g}$ cRNA. An equal amount of labeled cRNAs from each sample was then hybridized using the Agilent Gene Expression Hybridization kit (Agilent Technologies, Inc.) at $65^{\circ} \mathrm{C}$ for $17 \mathrm{~h}$.

Microarray data analysis. The acquired array images were analyzed using Agilent Feature Extraction software (version 11.0.1.1; Agilent Technologies, Inc.). Quantile normalization and subsequent data processing were performed using the GeneSpring GX v12.1 software package (Agilent Technologies, Inc.). The differential expression of IncRNAs or mRNAs with statistical significance between the two groups was screened by $\mathrm{P}$-value/False discovery rate (FDR). The $\mathrm{P}$-value was calculated using a t-test and modified according to the Benjamin Hochberg FDR method. The screening criteria were Ifold changel $\geq 2.0$ and FDR $<0.05$. The differentially expressed lncRNAs or mRNAs between the two samples were screened by fold-change (FC), and the screening criteria were $\mid \log _{2} \mathrm{FCl} \geq 1$ and $\mathrm{P}<0.05$. Gene Ontology (GO) and Kyoto Encyclopedia of Genes and Genomes (KEGG) pathway enrichment analyses were performed for the differentially expressed lncRNAs or mRNAs, as previously described (20).

Candidate IncRNA screening and RT-qPCR validation. From the microarray data, 14 lncRNAs were selected to perform RT-qPCR verification experiments with the array homologous plasma samples. The screening strategies were as follows: i) The top 5 gold level lncRNAs in the general list in the descending order of FC with the original expression $\geq 200$, namely LOC101927358, GAS5-AS1, LOC100507156, RP11-539G18.2 and ARHGEF 26-AS1; ii) lncRNAs with $\mid \mathrm{FCl} \geq 5$ and original expression $\geq 200$ in the sub-category analysis list with tissue-specific set to head-neck, disease-specific set to cancer, bio-process set to metastasis, namely CTD-2008L17.1 and LINC01539; iii) lncRNAs with $\mid \mathrm{FCl} \geq 5$ and co-existing in the general list and the differentially expressed lncRNA list in OPL based on SAGE (21), namely LINC00665 and NEAT1; iv) lncRNAs with $\mid \mathrm{FCl} \geq 10$ and co-existing in the general list and the differentially expressed lncRNA list in OSCC based on the microarray (20), namely $R P 11-250 B 2.3$ and $A P 001347.6$; and v) the top 3 lncRNAs with the largest FC coexisting in the general list and the tumor-related lncRNA list downloaded from the Lnc2Cancer database (http://www.bio-bigdata.com/lnc2cancer/down.jsp), namely HOTAIR_4, BCAR4 and MNXI-ASI. 
The primers were designed using primer premier 5.0 software (Premier Biosoft International) (Table SIII). The reverse transcription of total RNA was performed in a $20 \mu \mathrm{l}$ volume containing $500 \mathrm{ng}$ total RNA, $1 \mu \mathrm{l} 10 \mu \mathrm{M}$ primers, $1.6 \mu \mathrm{l}$ of $2.5 \mathrm{mM}$ dNTPs mixture, $4 \mu 1$ 5X First-Strand Buffer, $1 \mu 1$ 0.1 M DTT, $0.3 \mu \mathrm{l}$ RNase inhibitor, $0.2 \mu \mathrm{l}$ SuperScript III RT (Invitrogen; Thermo Fisher Scientific, Inc.) and $14.5 \mu \mathrm{l}$ water. The program was as follows: $50^{\circ} \mathrm{C}$ for $60 \mathrm{~min}, 70^{\circ} \mathrm{C}$ for $5 \mathrm{~min}$, and $4^{\circ} \mathrm{C}$ hold. The ViiA 7 Real-time PCR System (Applied Biosystems; Thermo Fisher Scientific, Inc.) was used for the RT-qPCR assay. A total of $2 \mu \mathrm{l}$ of the cDNA product was used as a template in $10 \mu 1$ reaction on a 384-well plate containing $5 \mu \mathrm{l}$ of $2 \mathrm{X}$ PCR master mix (Arraystar), $1 \mu \mathrm{l}$ of $10 \mu \mathrm{M}$ specific primer, $2 \mu \mathrm{l}$ of RNase-free water. The conditions were as follows: A denaturation step for $10 \mathrm{~min}$ at $95^{\circ} \mathrm{C}$, followed by 40 cycles of $10 \mathrm{sec}$ at $95^{\circ} \mathrm{C}$ and $60 \mathrm{sec}$ at $60^{\circ} \mathrm{C}$. Following amplification, the operation of the instrument was performed according to the procedure $\left(95^{\circ} \mathrm{C}, 10 \mathrm{sec} ; 60^{\circ} \mathrm{C}, 60 \mathrm{sec} ; 95^{\circ} \mathrm{C}\right.$, $15 \mathrm{sec}$ ) and slowly heated from $60^{\circ} \mathrm{C}$ to $99^{\circ} \mathrm{C}$ (-Ramp Rate was $0.05^{\circ} \mathrm{C} / \mathrm{sec}$ ). Each experiment was repeated in triplicate. The housekeeping gene used was $\beta$-actin. The $2^{-\Delta \Delta \mathrm{Cq}}$ method was used to measure relative expression levels (RELs) (22).

Target lncRNA screening and $R T-q P C R$ validation. A total of 4 lncRNAs were selected to be measured and validated by RT-qPCR in a large cohort. The screening strategies were as follows: i) The two lncRNAs with the top FC among the aforementioned 14 lncRNAs, namely ENST00000412740 and ENST00000588803; ii) the lncRNA with the largest FC in the general list, namely $N R \_038323$; and iii) the key lncRNA in OPL, namely $N R_{-}$131012. The RT-qPCR procedure was the same as that described earlier, and the primers used are listed in Table SIII.

Statistical analysis and evaluation of the 4 IncRNAs as diagnostic markers for the early diagnosis and staging of OSCC. The relative expression of lncRNAs was calculated using the $2^{-\Delta \Delta \mathrm{Cq}}$ method, where $\Delta \mathrm{Ct}=\mathrm{Ct}$ (target gene)-Ct ( $\beta$-actin), $\Delta \Delta \mathrm{Ct}=\Delta \mathrm{Ct}$ (experiment sample)- $\Delta \mathrm{Ct}$ (control sample). R language (version 3.3.2, https://www.r-project.org/) was used for data processing. The Shapiro-Wilk test was used to assess the normality of distribution and the Levene test (two sides) was used to assess the homogeneity of variance. If the data were normally distributed, analysis of variance was used to perform variance analysis among groups and the Tukey's HSD test was used to determine significant differences between groups. If the data were not normally distributed, the Kruskal-Wallis test rank sum test was used among groups, and the Dunn's test was used for post-hoc analysis. The Benjamin-Hochberg correction was performed to determine the P-values among groups.

Receiver operating characteristic (ROC) curve analysis and the area under the ROC curve (AUC) were used to evaluate the sensitivity and specificity of lncRNAs as novel diagnostic tools for the early diagnosis and staging of OSCC. A ROC curve was drawn using the ROC package in $\mathrm{R}$ language, and the comparison of AUC was performed using the DeLong test. Firth's Bias-Reduced Logistic Regression analysis was performed using the logistf package of $\mathrm{R}$ language, and variables were screened by the stepwise optimization method to determine the lncRNA combination with a high diagnostic efficiency. All tests were two-sided and $\mathrm{P}<0.05$ was considered to indicate a statistically significant difference.

\section{Results}

Differentially expressed profiles of IncRNAs and mRNAs in the plasma of patients with OSCC. Following image acquisition and data analysis, the expression matrices of lncRNAs and mRNAs were obtained. The volcano plot indicated that several lncRNAs and mRNAs were differentially expressed between the OSCC and normal samples (Fig. 1A and B). According to the screening standard, a total of 6,606 lncRNAs and 4,196 mRNAs were differentially expressed in the plasma of patients with OSCC. Furthermore, 3,511 lncRNAs and 1,766 mRNAs were upregulated, and 3,095 $\mathrm{lncRNAs}$ and 2,430 mRNAs were downregulated. The top 20 dysregulated 1 ncRNAs and mRNAs are presented in Tables SIV and SV, respectively. Hierarchical clustering analysis revealed that the expression profiles exhibited a good clustering effect on OSCC and normal plasma (Fig. 1C and D). The results of GO and KEGG analyses are presented in Tables SVI-SIX. The data have been deposited in NCBI's Gene Expression Omnibus and are accessible through GEO series accession no. GSE97251 (https://www.ncbi.nlm. nih.gov/geo/query/acc.cgi?acc=GSE97251).

Validation of candidate lncRNAs by RT-qPCR. Compared with the $\mathrm{H}$ group, 14 candidate IncRNAs were all differentially expressed in OSCC (Table I). Apart from NR_024050, which was downregulated (no statistical significance), the remaining 13 lncRNAs were all upregulated, and the FC value of 9 lncRNAs exhibited a statistically significant difference. These results were consistent with those of the microarray.

Validation of target lncRNAs by RT-qPCR. ENST00000412740, NR_131012, ENST00000588803 and NR_038323 were differentially expressed among the H, OPL, ESCC and ASCC groups $(\mathrm{P}<0.05)$. Furthermore, the differential expression of 4 target lncRNAs was compared between groups (Fig. 2). Compared with the H group, ENST00000412740, NR_131012, ENST00000588803 and NR_038323 were downregulated in the OPL group, and notably, they were upregulated in the ASCC group compared with the OPL group $(\mathrm{P}<0.05)$. Compared with the H group, NR_038323 was downregulated in the ESCC and ASCC groups $(\mathrm{P}<0.05)$. Compared with the OPL group, $N R \_131012$ was upregulated in the ESCC group $(\mathrm{P}<0.05)$. Compared with the ESCC group, ENST00000412740 and ENST00000588803 were upregulated in the ASCC group $(\mathrm{P}<0.05)$.

Screening of diagnostic combination and evaluation of the diagnostic efficacy of the 4 target lncRNAs. ROC curve analysis revealed that the 4 target lncRNAs exhibited excellent discriminative ability for OPL vs. H, OSCC vs. OPL and ASCC vs. ESCC, with an AUC >0.7, apart from NR_131012 and $N R \_038323$, which were considered as having moderate discriminative ability only for ASCC vs. ESCC, with an AUC of 0.558 (95\% CI, 0.0418-0.698) and 0.590 (95\% CI, 0.451-0.728), respectively (Fig. 3). However, they exhibited no discriminative ability for OSCC vs. H, apart from NR_038323 with an AUC >0.7 (Fig. S1). The logistic regression model 

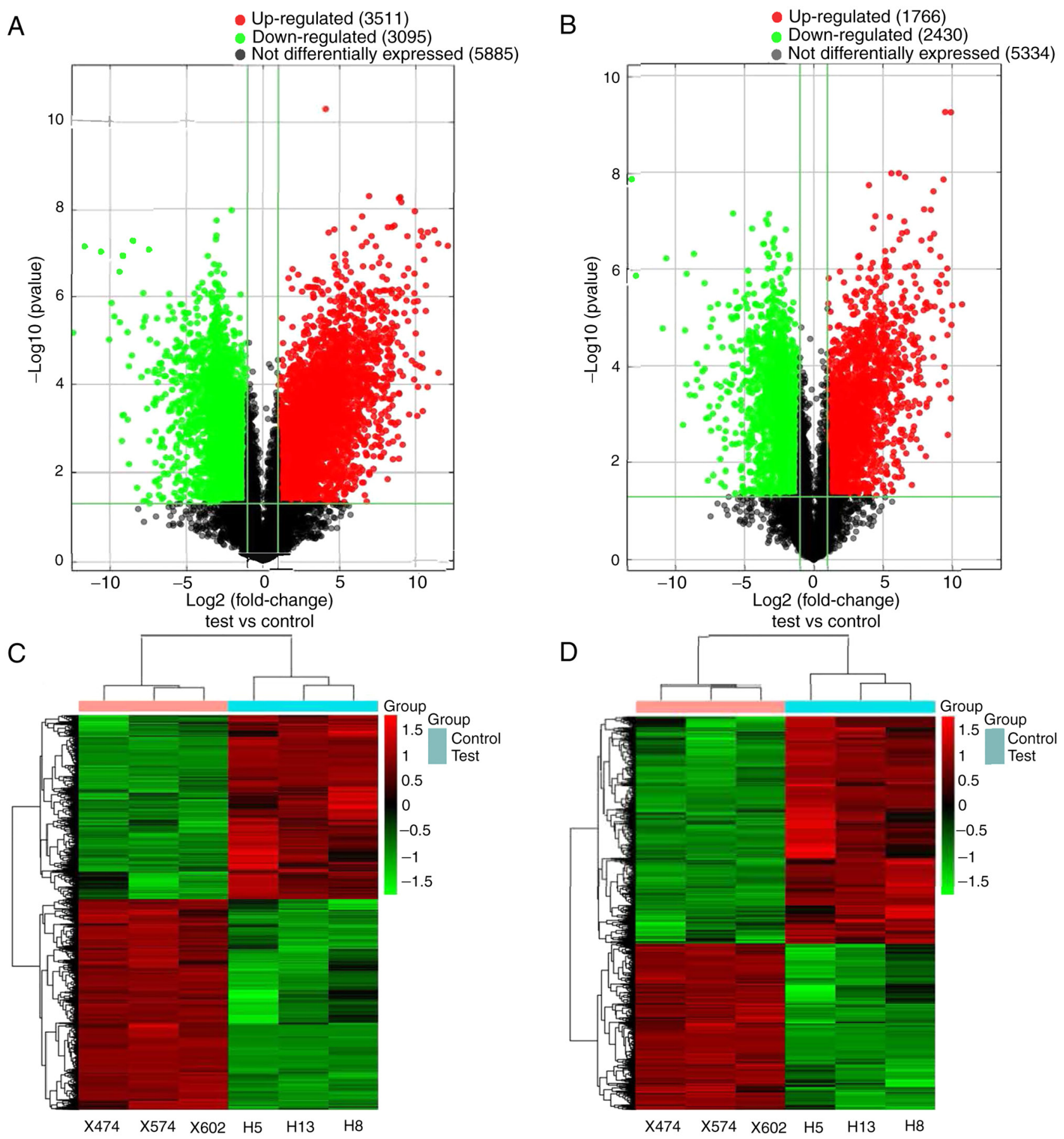

Figure 1. Volcano plot and hierarchical clustering analysis of differentially expressed (A and C) lncRNAs and (B and D) mRNAs between OSCC and normal plasma. The $\mathrm{x}$-axis and $\mathrm{y}$-axis in the volcano plot represent $\log _{2}(\mathrm{fold}$ change $)$ and $-\log _{10}(\mathrm{P}$-value), respectively. The red dots represent upregulated genes and the green dots represent downregulated genes. For hierarchical clustering, clusters of samples and genes are hierarchically clustered. Red color indicates that the expression level was increased. Green color indicates that the expression level was decreased. IncRNAs, long non-coding RNAs; OSCC, oral squamous cell carcinoma.

revealed that the combined lncRNAs provided a more prominent diagnostic efficacy than a single lncRNA, particularly for ASCC vs. ESCC (Table II). The sensitivity, specificity and cut-off value of each combination of lncRNAs are illustrated in Fig. 4.

\section{Discussion}

To the best of our knowledge, the present study was the first to identify the differential expression profiles of plasma lncRNAs in OSCC by microarray analysis. The reliability of microarray and quality of the array samples were verified to be credible by RT-qPCR using the array homologous samples. The results revealed that the profile of IncRNAs in plasma from patients with OSCC differed significantly from that of the healthy controls. The majority of the differentially expressed genes have been proven to be involved in the biological process of OSCC by GO and KEGG analyses $(20,21)$. However, there are only limited studies available on the diagnostic role of circulating lncRNAs in OSCC (23).

In the present study cohort, patients with TNM I/II stage OSCC accounted for $39.58 \%$ of primary OSCC cases, which 
Table I. REL and FC of 14 candidate lncRNAs (OSCC/H).

\begin{tabular}{|c|c|c|c|c|c|c|}
\hline $\operatorname{lncRNA}$ & OSCC & $\mathrm{H}$ & FP & P-value & FM & FDR \\
\hline ENST00000412740 & $3.94 \mathrm{E}-02$ & 5.39E-03 & 7.30 & 0.000066 & 1.22 & 0.025648541 \\
\hline ENST00000427048 & $4.02 \mathrm{E}-02$ & $2.62 \mathrm{E}-02$ & 1.54 & 0.064870 & 1.50 & 0.009596388 \\
\hline ENST00000428809 & $2.35 \mathrm{E}-02$ & $9.32 \mathrm{E}-03$ & 2.52 & 0.046177 & 2.81 & 0.011765579 \\
\hline ENST00000533736 & $3.72 \mathrm{E}-02$ & $1.25 \mathrm{E}-02$ & 2.98 & 0.000137 & 3.39 & 0.00325342 \\
\hline ENST00000588803 & $2.93 \mathrm{E}-02$ & 7.37E-03 & 3.98 & 0.000044 & 2.61 & 0.003797829 \\
\hline$N R \_024050$ & $4.88 \mathrm{E}-04$ & $6.88 \mathrm{E}-04$ & 0.71 & 0.151403 & 3.90 & 0.022136802 \\
\hline NR_037605 & $2.57 \mathrm{E}-03$ & $1.50 \mathrm{E}-03$ & 1.72 & 0.093041 & 3.22 & 0.001056383 \\
\hline NR_037901 & $9.75 \mathrm{E}-02$ & $3.57 \mathrm{E}-02$ & 2.73 & 0.000002 & 3.71 & 0.002373187 \\
\hline NR_038323 & 4.83E-02 & 1.33E-02 & 3.62 & 0.000002 & 5.30 & 0.000625734 \\
\hline NR_038835 & $6.38 \mathrm{E}-02$ & $1.79 \mathrm{E}-02$ & 3.56 & 0.000004 & 4.64 & 0.002772666 \\
\hline$N R \_040026$ & $9.28 \mathrm{E}-03$ & $2.40 \mathrm{E}-03$ & 3.87 & 0.000038 & 1.30 & 0.000843384 \\
\hline$N R \_121182$ & 8.62E-04 & 6.81E-04 & 1.26 & 0.582889 & 3.64 & 0.004240601 \\
\hline$N R \_131012$ & $3.06 \mathrm{E}-02$ & $9.75 \mathrm{E}-03$ & 3.14 & 0.012689 & 1.71 & 0.000283274 \\
\hline ис02 1qyj.1 & $1.13 \mathrm{E}-02$ & $9.76 \mathrm{E}-03$ & 1.15 & 0.651841 & 1.66 & 0.000092149 \\
\hline
\end{tabular}

REL, relative expression level; FC, fold-change; lncRNAs, long non-coding RNAs; OSCC, oral squamous cell carcinoma group; H, healthy control; FP, normalized fold-change of lncRNAs by RT-qPCR; FM, fold-change of lncRNAs by microarray; FDR, false discovery rate.

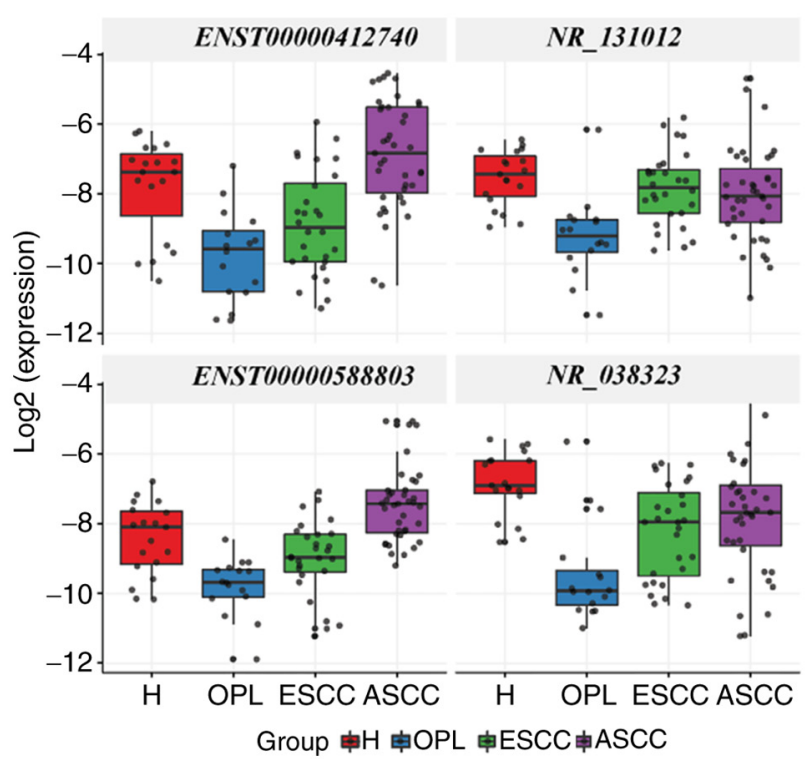

Figure 2. Boxplot of $\log _{2}$-transformed expression of ENST00000412740, NR_131012, ENST00000588803 and NR_038323 in the H, OPL, ESCC and ASCC groups. H, healthy controls; OPL, oral premalignant lesion; ESCC, early-stage squamous cell carcinoma; ASCC, advanced-stage squamous cell carcinoma.

was $22 \%$ of those involving the posterior third of the tongue reported in the literature (24), indicating that the early diagnosis of OSCC remains relatively low. However, patients with TNM I/II stage OSCC accounted for $47.37 \%$ of recurrent OSCC cases, which was slightly higher than that of primary OSCC, which may be associated with regular follow-up after surgery. A specialist may improve the early diagnosis of OSCC; however, this remains insufficient. The early diagnosis and staging of OSCC may aid doctors in determining effective and appropriate treatment strategies, including the scope of surgery, radiotherapy, chemotherapy and other adjuvant therapy, which has an important impact on the quality of life and prognosis of patients. These decisions are largely dependent on the clinical experience and subjective judgment of doctors; however, the lack of objective indicators leads to a bias in the making of these decisions. Therefore, an objective, accurate and minimally invasive biomarker is urgently required.

To date, $>1,000$ lncRNAs have been proven to be involved in various biological processes, and an increasing number of studies have demonstrated that plasma lncRNAs have great potential for use in tumor diagnosis, prognosis and in the evaluation of the therapeutic effects $(14,25-29)$. Circulating lncRNAs are derived from apoptosis, necrotic tissue and the active secretion of cells and lysis of circulating cells. Endogenous circulating lncRNAs are bound with proteins, which may be stable at room temperature and may endure multiple cycles of freezing and thawing (30,31). According to Schlosser et al (32), the level of lncRNAs in plasma has a certain association with its level in tissues, and lncRNAs may partly be derived from tissues. In the present study, when target lncRNAs were screened, the profiles of plasma lncRNAs and tissue lncRNAs in OSCC were compared and it was identified that only some of the differentially expressed lncRNAs was the same, which also indicated that the differentially expressed lncRNAs in the plasma were derived partly from tumor tissues. The expression of lncRNAs is tissue-specific (32-34). Therefore, the analysis of plasma lncRNA expression levels may be used as a minimally invasive diagnostic method for diseases.

In the present study, the four target lncRNAs were significantly downregulated in the plasma of patients with OPLs and gradually increased with the malignant transformation process. The differential expression of these four lncRNAs in different stages of OSCC indicated that they had the potential to be used as diagnostic markers for OPL and OSCC staging. The single lncRNAs ENST00000412740, NR_131012, ENST00000588803 or NR_038323 may distinguish OPL 
Table II. The results of Delong test of receiver operating characteristic curve for the combination of lncRNAs.

\begin{tabular}{lcll}
\hline Comparison & D & Group & P-value \\
\hline Combind2 vs. NR_038323 & 2.515717 & OSCC vs. OPL & 0.011879 \\
Combind3 vs. NR_131012 & 4.764378 & ASCC vs. ESCC & 0.000002 \\
Combind3 vs. NR_038323 & 4.631511 & ASCC vs. ESCC & 0.000004 \\
Combind3 vs. ENST00000588803 & 2.027116 & ASCC vs. ESCC & 0.042651 \\
Combind3 vs. ENST00000412740 & 2.677019 & ASCC vs. ESCC & 0.007428
\end{tabular}

lncRNAs, long non-coding RNAs; Combind2, combination of ENST00000588803 and NR_131012; Combind3, combination of ENST00000588803,ENST00000412740 and NR_131012; D, Delong test statistic; ASCC, advanced oral squamous cell carcinoma (TNM III/IV); ESCC, early oral squamous cell carcinoma (TNM I/II); OSCC, oral squamous cell carcinoma; OPL, oral premalignant lesions.
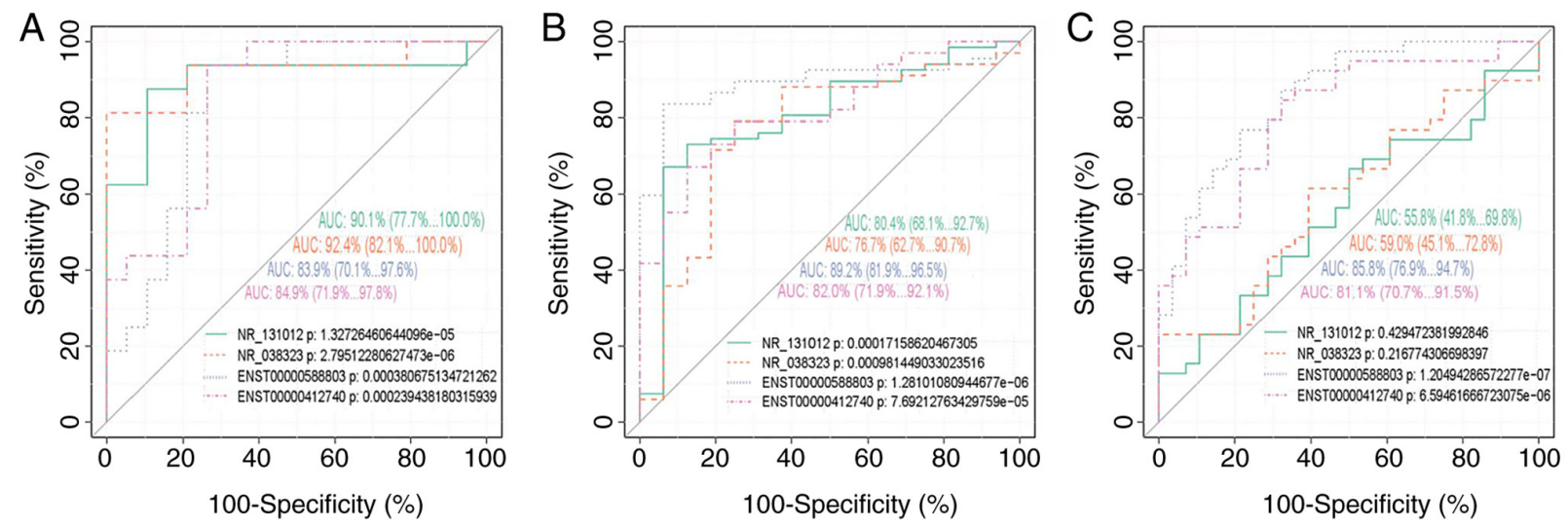

Figure 3. ROC curve analysis of single lncRNAs. ROC curve for (A) OPL vs. H, (B) OSCC vs. OPL and (C) ASCC vs. ESCC. AUC values are presented in the graphs. ROC, receiver operating characteristic; lncRNAs, long non-coding RNAs; OSCC, oral squamous cell carcinoma; OPL, oral premalignant lesion; ESCC, early-stage squamous cell carcinoma; ASCC, advanced-stage squamous cell carcinoma; AUC, area under the ROC curve.
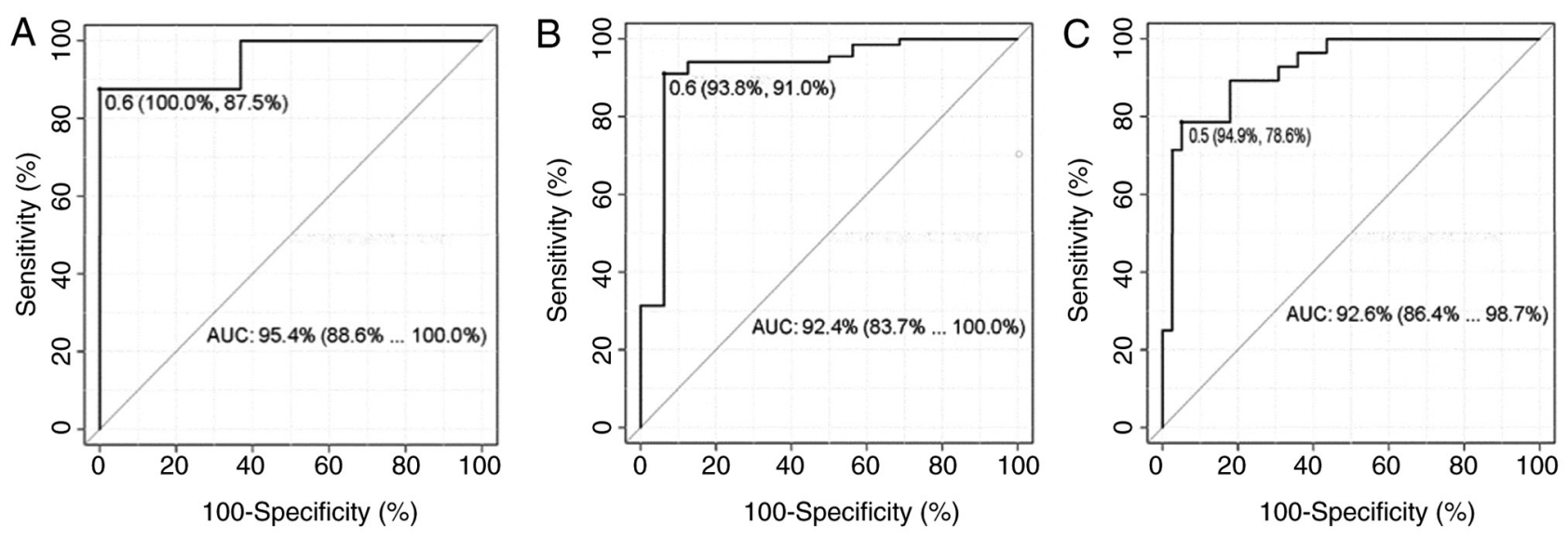

Figure 4. ROC curve analysis of combinations of 1ncRNAs. ROC curve of (A) ENST00000588803 combined with NR_038323 for OPL vs. H, (B) ENST00000588803 combined with NR_131012 for OSCC vs. OPL and (C) ENST00000588803 combined with ENST00000412740 and NR_131012 for ASCC vs. ESCC. AUC values are presented on the graphs. ROC, receiver operating characteristic; lncRNAs, long non-coding RNAs; OSCC, oral squamous cell carcinoma; OPL, oral premalignant lesion; ESCC, early-stage squamous cell carcinoma; ASCC, advanced-stage squamous cell carcinoma; AUC, area under the curve.

from the healthy controls, with an AUC of 0.901, 0.924, 0.839 and 0.849 , respectively, but was not effective for the determination of OSCC stage. To further prove the efficacy of the four lncRNAs for the diagnosis of OPLs and OSCC, ROC curve and logistic regression analyses were performed with optimal combinations. The results revealed that the AUCs of the combined lncRNAs were generally larger than those of single lncRNAs in distinguishing OSCC and OPLs, with a high sensitivity (93.8\%) and specificity (91.0\%), particularly in distinguishing ESCC from ASCC more effectively than all single lncRNAs with a high sensitivity (94.9\%) and specificity (78.6\%). The sensitivity of all combinations was far greater 
than that of the most well-known available biomarker, SCCA, with a sensitivity of $38.1 \%$ (35). Therefore, they may be very promising biomarkers for the early diagnosis and staging of OSCC. However, the expression levels of four lncRNAs in the ESCC group were similar to those of the H group; therefore, the dynamic monitoring of lncRNAs needs to combined with clinical examinations to distinguish the difference between the $\mathrm{H}$ group and ESCC group.

NEAT1 (NR_131012) is essential for the assembly and structural integrity of nuclear subunit paraspeckles (36) and is regulated by TP53. $p 53$ and $p R b$ pathway disruptions are an important step in the early stage of oral carcinogenesis (37), which may lead to the immortalization of oral epithelial cells (38). Among these, p53 is the 'guardian' of genome integrity, which has been found to upregulate NEAT1 expression. In oral premalignant lesions, TP53 mutation damages the $p 53$ signaling pathway (39) and the expression of NEAT1 is downregulated. With the malignant transformation process of cells, $p 53$ becomes activated under the effects of replication stress to upregulate NEATI expression, which promotes the formation of nuclear paraspeckles and the growth of highly divided cancer cells. Furthermore, NEAT1 promotes ATR signaling in response to replication stress to inhibit replication-related DNA damage and $p 53$ activation, thereby forming a negative feedback loop that attenuates the activation of $p 53$ in cells with DNA damage (40). This indicates that NEAT1 is downregulated in OPL and upregulated in ESCC and is expressed in ASCC. NEAT1 is highly expressed in various types of cancer, and its expression is associated with tumor size, TNM stage and distant metastasis; it is also a risk factor for a shorter overall survival (41). However, to the best of our knowledge, there are no studies available to date on the molecular mechanisms of the other 3 lncRNAs.

However, the exact mechanisms of these lncRNAs in the occurrence and development of OSCC remain unclear and cytological experiments are required to verify their functions. In addition, the sample size of the present study was small. For cross-sectional analysis, the validation sample needs to be further expanded, and the prognosis of patients requires follow-up, in order to make a comprehensive and accurate assessment of the clinical value of lncRNAs as diagnostic markers.

In conclusion, the present study demonstrated that the expression profiles of plasma lncRNAs are altered in OSCC compared with normal controls. ENST00000412740, NR_131012, ENST00000588803 and NR_038323 were differentially expressed in different stages of OSCC and their expression became altered with the malignant progression of OSCC. This suggests that these four lncRNAs may be promising biomarkers for the early diagnosis and staging of OSCC. Furthermore, the diagnostic efficacy of the combined lncRNAs was more prominent than that of a single lncRNA.

\section{Acknowledgements}

The authors would like to thank Professor Xiaofei Tang and Professor Xinyan Zhang (Institute of Stomatology, Capital Medical University) for their suggestions and comments, Professor Zhengxue Han, Dr Yao Liu and Dr Meihua Zhang (Beijing Stomatological Hospital, Capital Medical University) for their help in sample collection, and Mr Zhigang Wang
(Medical Data Processing Center, Peking Union Medical College Hospital) for data processing.

\section{Funding}

The present study was supported by a grant from the National Natural Science Foundation of China (grant no. 81372897).

\section{Availability of data and materials}

The datasets used and/or analyzed during the present study are available from the corresponding author upon reasonable request.

\section{Authors' contributions}

HJ and XW acquired the data, performed the experiments and drafted the initial manuscript. HJ, XW and ZS designed the experiments, interpreted the data and analyzed the results. SZ and $\mathrm{HJ}$ revised and approved the final version of the manuscript. All authors have read and approved the final manuscript, and agreed to be accountable for all aspects of the research in ensuring that the accuracy or integrity of any part of the work are appropriately investigated and resolved.

\section{Ethics approval and consent to participate}

The present study was approved by the Ethics Committee of the Capital Medical University Beijing Stomatological Hospital (Beijing, China; approval no. 201314) and written informed consent was provided by all participants prior to the study start.

\section{Patient consent for publication}

Not applicable.

\section{Competing interests}

The authors declare that they have no competing interests.

\section{References}

1. Gupta B, Johnson NW and Kumar N: Global epidemiology of head and neck cancers: A continuing challenge. Oncology 91: 13-23, 2016.

2. Bray F, Ferlay J, Soerjomataram I, Siegel RL, Torre LA and Jemal A: Global cancer statistics 2018: GLOBOCAN estimates of incidence and mortality worldwide for 36 cancers in 185 countries. CA Cancer J Clin 68: 394-424, 2018.

3. Sinevici N and O'sullivan J: Oral cancer: Deregulated molecular events and their use as biomarkers. Oral Oncol 61: 12-18, 2016.

4. Ghani WMN, Ramanathan A, Prime SS, Yang YH, Razak IA, Abdul Rahman ZA, Abraham MT, Mustafa WMW, Tay KK, Kallarakkal TG, et al: Survival of oral cancer patients in different ethnicities. Cancer Invest 37: 275-287, 2019.

5. Alonso JE, Han AY, Kuan EC, Strohl M, Clai JM, St John MA, Ryan WR and Heaton CM: The survival impact of surgical therapy in squamous cell carcinoma of the hard palate. Laryngoscope 128: 2050-2055, 2018.

6. Bloebaum M, Poort L, Bockmann R and Kessler P: Survival after curative surgical treatment for primary oral squamous cell carcinoma. J Craniomaxillofac Surg 42: 1572-1576, 2014.

7. Quinn JJ and Chang HY: Unique features of long non-coding RNA biogenesis and function. Nat Rev Genet 17: 47-62, 2016. 
8. Kornienk AE, Guenzl PM, Barlow DP and Pauler FM: Gene regulation by the act of long non-coding RNA transcription. BMC Biol 11: 59, 2013.

9. Geisler S and Coller J: RNA in unexpected places: Long non-coding RNA functions in diverse cellular contexts. Nat Rev Mol Cell Biol 14: 699-712, 2013.

10. Wapinski $\mathrm{O}$ and Chang HY: Long noncoding RNAs and human disease. Trends Cell Biol 21: 354-361, 2011.

11. Lalevee S and Fei R: Long noncoding RNAs in human disease: Emerging mechanisms and therapeutic strategies. Epigenomics 7: 877-879, 2015

12. Chandra Gupta S and Nandan Tripathi Y: Potential of long non-coding RNAs in cancer patients: From biomarkers to therapeutic targets. Int J Cancer 140: 1955-1967, 2017.

13. Zhang L, Meng X, Zhu XW, Yang DC, Chen R, Jiang Y and $\mathrm{Xu}$ T: Long non-coding RNAs in Oral squamous cell carcinoma: Biologic function, mechanisms and clinical implications Mol Cancer 18: 102, 2019.

14. Ren S, Wang F, Shen J, Sun Y, Xu W, Lu J, Wei M, Xu C, Wu C Zhang $\mathrm{Z}$, et al: Long non-coding RNA metastasis associated in lung adenocarcinoma transcript 1 derived miniRNA as a novel plasma-based biomarker for diagnosing prostate cancer. Eur J Cancer 49: 2949-2959, 2013.

15. Kogo R, Shimamura T, Mimori K, Kawahara K, Imoto S, Sudo T, Tanaka F, Shibata K, Suzuki A, Komune S, et al: Long noncoding RNA HOTAIR regulates polycomb-dependent chromatin modification and is associated with poor prognosis in colorectal cancers. Cancer Res 71: 6320-6326, 2011.

16. Sun M, Jin FY, Xia R, Kong R, Li JH, Xu TP, Liu YW, Zhang EB, Liu XH and De W: Decreased expression of long noncoding RNA GAS5 indicates a poor prognosis and promotes cell proliferation in gastric cancer. BMC Cancer 14: 319, 2014.

17. Gibb EA, Enfield KS, Stewart GL, Lonergan KM, Chari R, Ng RT, Zhang L, MacAulay CE, Rosin MP and Lam WL: Long non-coding RNAs are expressed in oral mucosa and altered in oral premalignant lesions. Oral Oncol 47: 1055-1061, 2011.

18. Tang $\mathrm{H}, \mathrm{Wu} \mathrm{Z}$, Zhang J and Su B: Salivary lncRNA as a potential marker for oral squamous cell carcinoma diagnosis. Mol Med Rep 7: 761-766, 2013.

19. Fang Z, Wu L, Wang L, Yang Y, Meng Y and Yang HL: Increased expression of the long non-coding RNA UCA1 in tongue squamous cell carcinomas: A possible correlation with cancer metastasis Oral Surg Oral Med Oral Pathol Oral Radiol 117: 89-95, 2014.

20. Jia H, Wang $X$ and Sun Z: Exploring the long noncoding RNAs-based biomarkers and pathogenesis of malignant transformation from dysplasia to oral squamous cell carcinoma by bioinformatics method. Eur J Cancer Prev 29: 174-181, 2020.

21. Jia H, Wang X and Sun Z: Exploring the molecular pathogenesis and biomarkers of high risk oral premalignant lesions on the basis of long noncoding RNA expression profiling by serial analysis of gene expression. Eur J Cancer Prev 27: 370-378, 2018.

22. Livak KJ and Schmittgen TD: Analysis of relative gene expression data using real-time quantitative PCR and the 2(-Delta Delta C(T)) method. Methods 25: 402-408, 2001.

23. Shao T, Huang J, Zheng Z, Wu Q, Liu T and Lv X: SCCA, TSGF, and the long non-coding RNA AC007271.3 are effective biomarkers for diagnosing oral squamous cell carcinoma. Cell Physiol Biochem 47: 26-38, 2018.

24. Sciubba JJ: Oral cancer: The importance of early diagnosis and treatment. Am J Clin Dermalol 2: 239-251, 2001.

25. Meseure D, Drak Alsibai K, Nicolas A, Bieche I and Morillon A: Long noncoding RNAs as architects in cancer epigenetics, prognostic biomarkers, and potential therapeutic targets. Biomed Res Int 2015: 320214, 2015.
26. Kladi-Skandali A, Michaelidou K, Scorilas A and Mavridis K Long noncoding rnas in digestive system malignancies: A novel class of cancer biomarkers and therapeutic targets? Gastroenterol Res Pract 2015: 319861, 2015.

27. Fatima R, Akhade VS, Pal D and Rao SM: Long noncoding RNAs in development and cancer: Potential biomarkers and therapeutic targets. Mol Cell Ther 3: 5, 2015.

28. Tang Q, Ni Z, Cheng Z, Xu J, Yu H and Yin P: Three circulating long non-coding RNAs act as biomarkers for predicting NSCLC. Cell Physiol Biochem 37: 1002-1009, 2015.

29. Li J, Wang X, Tang J, Jiang R, Zhang W, Ji J and Sun B: HULC and Linc00152 act as novel biomarkers in predicting diagnosis of hepatocellular carcinoma. Cell Physiol Biochem 37: 687-696, 2015.

30. Ge Q, Zhou Y, Lu J, Bai Y, Xie X and Lu Z: miRNA in plasma exosome is stable under different storage conditions. Molecules 19: 1568-1575, 2014.

31. Tsui NB, Ng EK and Lo YM: Stability of endogenous and added RNA in blood specimens, serum, and plasma. Clin Chem 48: 1647-1653, 2002

32. Schlosser K, Hanson K, Villeneuve PK, Dimitroulakos J, McIntyre L, Pilote L and Stewart DJ: Assessment of circulating LncRNAs under physiologic and pathologic conditions in humans reveals potential limitations as biomarkers. Sci Rep 6: 36596, 2016.

33. Mercer TR, Gerhardt DJ, Dinger ME, Crawford J, Trapnell C, Jeddeloh JA, Mattick JS and Rinn JL: Targeted RNA sequencing reveals the deep complexity of the human transcriptome. Nat Biotechnol 30: 99-104, 2011.

34. Prensner JR, Iyer MK, Sahu A, Asangani IA, Cao Q, Patel L, Vergara IA, Davicioni E, Erho N, Ghadessi M, et al: The long noncoding RNA SChLAPI promotes aggressive prostate cancer and antagonizes the SWI/SNF complex. Nat Genet 45: 1392-1298, 2013

35. Kurokawa H, Yamashita Y, Tokudome S and Kajiyama M: Combination assay for tumor markers in oral squamous cell carcinoma. J Oral Maxillofac Surg 55: 964-966, 1997.

36. Clemson CM, Hutchinson JN, Sara SA, Ensminger AW, Fox AH, Chess A and Lawrence JB: An architectural role for a nuclear noncoding RNA: NEAT1 RNA is essential for the structure of paraspeckles. Mol cell 33: 717-726, 2009.

37. Leemans CR, Braakhuis BJ and Brakenhoff RH: The molecular biology of head and neck cancer. Nat Rev Cancer 11: 9-22, 2011.

38. Smeets SJ, van der Plas M, Schaaij-Visser TB, van Veen EA, van Meerloo J,Braakhuis BJ, Steenbergen RD and Brakenhoff RH: Immortalization of oral keratinocytes by functional inactivation of the p53 and pRb pathways. Int J Cancer 128: 1596-1605, 2011.

39. Graveland AP, Bremmer JF, de Maaker M, Brink A, Cobussen P, Zwart M, Braakhuis BJ, Bloemena E, van der Waal I, Leemans CR and Brakenhoff RH: Molecular screening of oral precancer. Oral Oncol 49: 1129-1135, 2013.

40. Adriaens C, Standaert L, Barra J, Latil M, Verfaillie A, Kalev P, Boeckx B, Wijinhoven PW, Radaelli E, Vermi W, et al: p53 induces formation of NEAT1 lncRNA-containing paraspeckles that modulate replication stress response and chemosensitivity. Nat Med 22: 861-868, 2016.

41. Fang J, Qiao F, Tu J, Xu J, Ding F, Liu Y, Akuo BA, Hu J and Shao S: High expression of long non-coding RNA NEAT1 indicates poor prognosis of human cancer. Oncotarget 8: 45918-45927, 2017.

This work is licensed under a Creative Commons Attribution-NonCommercial-NoDerivatives 4.0 International (CC BY-NC-ND 4.0) License. 